\title{
PROCESOS COGNITIVOS Y SOCIO AFECTIVOS IMPLICADOS EN EL APRENDIZAJE DEL CANTO CORAL EN LA EDUCACIÓN PRIMARIA
}

\author{
PROCESSOS COGNITIVOS E SOCIOAFETIVOS ENVOLVIDOS NA \\ APRENDIZAGEM DO CANTO CORAL NA EDUCAÇÃO PRIMÁRIA
}

\section{COGNITIVE AND SOCIO-AFFECTIVE PROCESSES INVOLVED IN CORAL CORNER LEARNING IN PRIMARY EDUCATION}

\author{
Digna La Rosa \\ Universidad Pedagógica Experimental Libertador (UPEL) \\ María Margarita Villegas \\ Universidade Federal Rural do Semi Árido (UFERSA) \\ Fredy Enrique González \\ Universidade Federal do Rio Grande do Norte (UFRN)
}

\section{RESUMEN}

Este trabajo se propuso analizar los procesos cognitivos y socio-afectivos implicados en el aprendizaje del canto coral en niños de educación primaria en una escuela pública venezolana. El estudio se realizó con 7 niñas y 3 niños de $\operatorname{los} 4^{\circ}, 5^{\circ}$ y $6^{\circ}$ grados de educación primaria. Las técnicas de investigación fueron la observación participante, interrogatorios colectivos y la entrevista con apoyo en un cuestionario, grabaciones de audio y video; así mismo se usaron instrumentos musicales como el cuatro venezolano y el piano. El análisis de contenido de la información recolectada mediante las observaciones y los diálogos con los alumnos participantes en el estudio develan el uso tanto de procesos cognitivos (percepción, memoria, descripción, imitación y evaluación) como de procesos sociales (sentido de grupo, solidaridad, etc.) y afectivos (satisfacción, responsabilidad, constancia y respeto). El reconocimiento de la presencia de todos estos procesos proporcionó una mayor calidad a las actividades de enseñanza- aprendizaje del canto coral a estudiantes de educación básica. Además, haciendo las adaptaciones adecuadas, los resultados de esta pesquisa podrían ser utilizados en la educación musical en general en diversos niveles educativos.

PALABRAS CLAVE: Canto coral. Procesos cognitivos-afectivos. Aprendizaje. Educación musical.

\begin{tabular}{|l|l|l|l|l|l|} 
Revista RBBA & ISSN 2316-1205 & Vitória da Conquista & V.9 n ${ }^{\circ} 1$ & p. 263-289 & Julho/2020 \\
\hline
\end{tabular}




\title{
RESUMO
}

Este trabalho teve como objetivo analisar os processos cognitivos e socioafetivos envolvidos na aprendizagem do canto coral em crianças de escolas primárias de uma escola pública venezuelana. $\mathrm{O}$ estudo foi realizado com 7 meninas e 3 meninos da $4^{\mathrm{a}}, 5^{\mathrm{a}}$ e $6^{\mathrm{a}}$ séries do ensino fundamental. As técnicas de investigação foram a observação participante, interrogatórios coletivos e a entrevista com suporte em questionário, gravações em áudio e vídeo; da mesma forma, instrumentos musicais como o cuatro venezuelano e o piano foram usados. A análise de conteúdo das informações coletadas por meio de observações e diálogos com os alunos participantes do estudo revela o uso de processos cognitivos (percepção, memória, descrição, imitação e avaliação) e processos sociais (senso de grupo, solidariedade, etc.) e afetivos (satisfação, responsabilidade, perseverança e respeito). O reconhecimento da presença de todos esses processos proporcionou maior qualidade às atividades de ensino-aprendizagem do canto coral aos alunos do ensino fundamental. Além disso, ao fazer as devidas adaptações, os resultados desta pesquisa podem ser utilizados no ensino da música em geral em vários níveis educacionais.

PALAVRAS-CHAVE: Canto coral. Processos cognitivo-afetivos. Aprendizagem. Educação musical.

\begin{abstract}
This work aimed to analyze the cognitive and socio-affective processes involved in the learning of choral singing in children from primary schools in a Venezuelan public school. The study was carried out with 7 girls and 3 boys from the 4th, 5th and 6th grades of elementary school. The investigative techniques were participant observation, collective interrogations and the interview supported by a questionnaire, audio and video recordings; similarly, musical instruments like the Venezuelan cuatro and the piano were used. The content analysis of the information collected through observations and dialogues with the study's participating students reveals the use of cognitive processes (perception, memory, description, imitation and evaluation) and social processes (sense of group, solidarity, etc.) and affective (satisfaction, responsibility, perseverance and respect). The recognition of the presence of all these processes offers higher quality to the teaching-learning activities of choral singing to elementary school students. In addition, when making the necessary adaptations, the results of this research can be used in the teaching of music in general at various educational levels.
\end{abstract}

KEYWORDS: Choral singing. Cognitive-affective processes. Learning. Musical education.

\section{Introducción}

El punto de partida del estudio reportado en este artículo lo constituyó la preocupación de la primera autora quien, como formadora de futuros profesores de Música, estudiantes de Educación Musical en una universidad pública venezolana de formación docente, apreció que éstos -al ingresar a la Universidad- carecen de los conocimientos y habilidades necesarias que 
permitan pronosticar que tendrán un buen desempeño académico en los estudios necesarios para graduarse como profesores de música.

En efecto, es frecuente que muchos de los estudiantes que ingresan a la Especialidad de Educación Musical en la mencionada universidad, lo hacen sin poseer los conocimientos musicales básicos que deberían haber adquirido, ni las habilidades que habrían tenido que desarrollar en los niveles educativos precedentes, como, por ejemplo, ser afinados al cantar con diferentes voces o líneas melódicas sin desentonar, leer arreglos musicales (partituras) para canto polifónico, entre otros. No obstante, en la universidad estas deficiencias no son subsanadas, porque la formación universitaria está orientada hacia el conocimiento y dominio de géneros musicales y poco se dedica a la metodología de enseñanza para el desarrollo de habilidades musicales en niveles iniciales. En ello incide mucho la creencia de que con tales habilidades se nace y que no se pueden desarrollar mediante procesos educativos adecuados. Esto afecta significantemente el desempeño pedagógico de quienes egresan de la universidad como profesores de música porque no están preparados para educar musicalmente a los estudiantes de las escuelas básicas públicas.

Esto sugiere la necesidad de revisar, no sólo el plan de estudios de la Especialidad de Educación Musical con el fin de adecuarlo a la realidad educativa con la que se enfrentarán los Profesores de Música que demanda no sólo los conocimientos y habilidades necesarias para la ejecución de algún instrumento sino además, y principalmente, requiere de un conocimiento idóneo de los fundamentos metodológicos para la educación musical porque quien enseña, “[...] no ha de hacerlo por inercia o por ensayo y error" (LUCATO, 2001, p. 4), sino basándose sobre unos fundamentos pedagógicos y psicológicos que le permitan diseñar estrategias tales que hagan altamente probable el aprendizaje de sus estudiantes. En esta dirección han sido desarrolladas algunas proposiciones, una de ellas es la de Rusinek (2003) quien propone reorganizar los procesos de enseñanza de la música apoyándose en la teoría de las inteligencias múltiples la cual ha contribuido a

[...] cambiar los puntos de vista tradicionales sobre la inteligencia humana excesivamente centrados en el Coeficiente Intelectual, abriendo nuevos espacios a la intervención psico-educativa, con la esperanza de ofrecer una educación de calidad y, sobre todo, de mejorar el funcionamiento cognitivo de los alumnos. (PÉREZ SÁNCHEZ; BELTRÁN LLERA, 2006, p. 148). 


\section{PROCESOS COGNITIVOS Y SOCIO AFECTIVOS IMPLICADOS EN EL APRENDIZAJE DEL CANTO CORAL EN LA EDUCACIÓN PRIMARIA}

Una de tales inteligencias es la Inteligencia Musical la cual tiene que ver con la forma como los seres humanos nos relacionamos con los sonidos y especialmente se vincula con la sensibilidad que tenemos con sus aspectos melódicos, armónicos, rítmicos, tímbricos y estructurales. Mas, como afirma Morán $(2009$, p. 4) "la música [va mucho] más allá de sus elementos acústicos". Esta misma autora, afirma que "cualquier intento por explicar la aptitud artística [en general y musical en particular] debe tomar en cuenta la organización funcional del cerebro, especialmente por las cuestiones de asimetría hemisférica y dominancia cerebral." (MORÁN, 2009, p.10). Más adelante, haciendo referencia a varias investigaciones sobre el impacto de la música sobre la estructura y el funcionamiento del cerebro, sostiene que

[...] las respuestas del cerebro pueden evolucionar de manera diferente en el transcurso de un año, según los niños hayan sido formados o no en el conocimiento y la experiencia musical. Estos cambios tienen una relación directa con las mejores habilidades cognitivas constatadas en los niños que practican la música, lo que constituye una evidencia de que el aprendizaje musical tiene un efecto positivo sobre la memoria y la atención. Los hallazgos anteriores indican la conveniencia de que la presencia y enseñanza musical sea parte medular en la formación integral del ser humano. No solamente por sus repercusiones en el desarrollo de competencias cognitivas y emocionales sino por la importancia intrínseca de la música en aspectos fisiológicos, individuales y sociales. (MORÁN, 2009, p. 10) (Subrayados añadidos).

Además de lo antes expuesto, es necesario reconocer la importancia de la dimensión cognitiva (VILLEGAS; GONZÁLEZ, 2003) en el aprendizaje musical y que los procesos cognitivos que intervienen en dicho aprendizaje "[...] son complejos y tienen lugar en las estructuras de la memoria implicadas en la construcción del conocimiento" (RODRÍGUEZ; FERNÁNDEZ, 1997; p. 96), por tal motivo, su enseñanza debe estimular el desarrollo tanto de los procesos afectivos, asociados con el goce estético de la música implicados en la producción de ritmos, melodías, armonías, manejo de la voz y expresión corporal (RUSINEK, 2003; GRINSPUN SIGUELNITZKY; POBLETE LAGOS, 2018), como de los procesos cognitivos que también son fundamentales en la creación musical. Así que una educación musical provechosa debe contribuir al desarrollo de habilidades de memoria, atención, percepción y discriminación, entre otros procesos cognitivos. Por tanto, si los profesores de música no poseen conocimientos sobre los procesos que son activados durante el aprendizaje musical, especialmente de los alumnos de educación básica, primaria o fundamental, desaprovechan la oportunidad para producir un impacto más significativo en ellos. 


\section{PROCESOS COGNITIVOS Y SOCIO AFECTIVOS IMPLICADOS EN EL APRENDIZAJE DEL CANTO CORAL EN LA EDUCACIÓN PRIMARIA}

Así que revalorizar las potencialidades que el aprendizaje de la música tiene para el desenvolvimiento pleno, tanto en lo cognitivo y afectivo como en lo social, de los estudiantes de la educación primaria es una necesidad. Es durante este periodo cuando los niños y las niñas tienen más y mejores oportunidades de desarrollar no solamente su sensibilidad, gusto y habilidades por y para la música sino también su sentido de pertenencia a un grupo social mayor, sea la familia, los vecinos, los compañeros de clase y, por fin, la sociedad en general.

En este último sentido, el aprendizaje musical, especialmente aquel en el que la música es construida multi-vocal o multi-instrumentalmente, es una estrategia adecuada para la superación del egocentrismo característico de las edades tempranas del desenvolvimiento social de los estudiantes de los primeros niveles educativos. (PEREIRA; VASCONCELOS, 2007)

En efecto, el egocentrismo es un fenómeno no sólo psicológico sino conductual que

[...] ocurre a una edad en que la educación encuentra las condiciones más favorables, el egocentrismo infantil es un tema igualmente relevante para la psicología y la pedagogía. No solo el orden de las ideas (egocentrismo lógico), sino sobre todo la validez del conocimiento (egocentrismo ontológico) sufrirá serias pérdidas, cuando esté condicionado por un fenómeno subjetivo e inconsciente que no permita al sujeto hacer la distinción necesaria entre su yo y la realidad externa que le rodea $\underline{Y \text {, dentro de una perspectiva de desarrollo }}$ integral y armónico, deteriorado el proceso cognitivo, todo lo demás se verá comprometido. Imagínense las consecuencias que puede tener un egocentrismo acentuado y tardío en los campos afectivo y moral, en los dominios del lenguaje y la comunicación social (diálogo y cooperación), etc. Problemas que incitan tanto al psicólogo como al educador. (FONZAR, 1986, p. 100). (Subrayado añadido)

A partir de lo expuesto puede inferirse que los profesores de música al egresar de la universidad se enfrentan a enormes desafíos para propiciar la educación musical de los estudiantes de la educación básica. ¿Cómo superarlos? Una de las opciones para formar musicalmente a los niños y las niñas de entre 6 y 12 años, previstas en el Sistema Educativo Bolivariano (2007) es el canto coral, este no sólo es una de las actividades artísticas musicales más accesibles dentro del ámbito escolar de las escuelas públicas, sino que puede ser asumido como recurso para promover tanto procesos cognitivos y afectivos individuales como la socialización de quienes participan del mismo por cuanto " [...] deben aprender a escuchar a sus compañeros, aprender a esperar, comenzar en el momento oportuno, con una dinámica, una frecuencia (tono), etc. "El hecho de cantar en una agrupación coral desarrolla un modelo educativo inclusivo de forma inherente [...]" (PEREZ-ALDEGUER, 2014, p. 392). No debe 


\section{PROCESOS COGNITIVOS Y SOCIO AFECTIVOS IMPLICADOS EN EL APRENDIZAJE DEL CANTO CORAL EN LA EDUCACIÓN PRIMARIA}

olvidarse que para aprender a cantar en coro se requiere de un trabajo sostenido y planeado. Por tanto, comprometerse con proyecto de ese tipo exige de mucha disciplina y coordinación de acciones, e “[...] implica un número de horas de práctica mayor al que podemos dedicarle durante las horas de clase dentro del recinto escolar" (LUCATO, 2001, p. 5), por lo cual va a requerir de tiempo extra fuera de la rutina de la escuela.

Con el fin de obtener evidencias que permitan constatar empíricamente los beneficios que en alumnos de educación básica, tiene el aprendizaje del canto coral en cuanto a expresividad, desenvolvimiento personal-social e interés por desarrollar y educar su propia voz musical, se llevó a cabo la investigación reportada en este artículo. Su viabilidad fue propiciada por el hecho de que la primera autora se desempeña como profesora de música en escuelas básicas públicas de zonas deprimidas ubicadas en el área de influencia de la universidad. La idea fue constatar si la participación de sus alumnos en grupos de música coral propiciaba cambios satisfactorios en ellos en las tres dimensiones ya indicadas: cognitiva, afectiva y social.

Así que, con la orientación y co-orientación de la segunda autora y del segundo autor, respectivamente, la primera autora emprendió un estudio basado en su propia práctica como directora de agrupaciones corales en las escuelas donde se desempeña como profesora de música. El trabajo pretendió, no sólo aprovechar el canto coral para desarrollar desde edades tempranas, sensibilidades, habilidades, y capacidades para hacer música junto con otros, sino también para identificar buenas prácticas en la enseñanza de la música que pudieran ser incorporadas a los planes de formación inicial de los futuros docentes de la Especialidad de Educación Musical en la universidad.

Dado el papel que juegan las ciencias cognitivas en la interpretación de procesos de aprendizaje (GONZÁLEZ, 1999), el interés principal del estudio fue identificar los procesos cognitivos y afectivos implicados en el aprendizaje del canto coral durante educación primaria y su incidencia en el desarrollo personal y social de los niños y niñas durante su educación primaria, especialmente de quienes están en situación de vulnerabilidad puesto que en estos contextos la música adquiere un notable valor como expresión de resiliencia (CABRELLES SAGREDO, 2007).

Las interrogantes orientadoras de la pesquisa fueron las siguientes ¿Cuáles procesos cognitivos y socio-afectivos ponen en juego los alumnos de educación primaria en escuelas públicas durante su aprendizaje de canto coral? ¿La concientización de esos procesos mejora 
sus habilidades musicales? Basados en estas interrogantes fueron formulados los siguientes objetivos:

- Analizar los procesos cognitivos implicados en el aprendizaje del canto coral en alumnos de educación primaria

- Caracterizar los procesos socio-afectivos que se desarrollan durante el aprendizaje del canto coral en la educación primaria.

\section{Referencias teóricas}

\section{El Canto Coral en la escuela}

De acuerdo con la Asociación Alavesa de Coros se llama coro "a un grupo de personas que interpretan una función de música vocal de manera simultánea y coordinada. Es la forma de interpretación colectiva de las obras cantadas o que necesitan la intervención de la voz." En un coro interviene un conjunto de personas que cantan simultáneamente una pieza concertada (CROWTHER, 2005). El canto coral es una práctica musical abierta a mucha más gente que otro tipo de prácticas musicales (MENDOZA, 2001). Es ese carácter colectivo de la música coral una de sus características que hacen que sea más viable su uso la escuela, siendo además una práctica que requiere principalmente de recursos humanos, por lo cual resulta accesible tanto al docente como a los estudiantes.

Es importante realizar la práctica del canto coral en las instituciones escolares porque, a la par que permite el desarrollo musical de los alumnos integrantes del coro, con todos los valores formativos y humanos que de la música se derivan, propicia su integración social, comunitaria y colectiva, (CROWTHER, 2005; SERRANO \& GIL, 2003).

Desde el punto de vista estrictamente musical, con la práctica coral en la escuela, se contribuye con la formación musical del alumno en la medida en que éste aprende a: cantar bien al unísono y en diferentes líneas melódicas a la vez; escuchar y ajustar el canto al de las otras voces o al acompañamiento; interpretar una variedad de estilos, aprender elementos de interpretación, desarrollar el oído interior, la memoria musical, el sentido rítmico y la técnica vocal (CROWTHER, 2005).

Como expresión oral-musical, de acuerdo con Galdón (2013), existen varios tipos de canto coral: a) monofónico o monódico, en el cual sólo existe una línea melódica, cantada por todo el grupo en el mismo tono o altura; b) homofónico, música compuesta por voces que 
evolucionan al mismo ritmo, como una melodía con cada nota armonizada; c) polifónico, en el cual encontramos varias voces, varias partes, en donde cada una de ellas conserva su independencia, a la par que está sujeta armónicamente a las restantes. el uso y práctica de este tercer tipo depende de la experiencia del grupo coral, de la madurez de los participantes en el aspecto musical, de la edad de los integrantes del coro o, en todo caso de los objetivos que persigue el director del coro.

Por tanto, para formar en el canto coral a los alumnos de la educación básica, es necesario conocer su nivel de desarrollo; se recomienda empezar con los cantos monofónicos, hasta que se adquiera la seguridad necesaria, luego seguir con líneas polifónicas sencillas como el canon, para que cada alumno pueda educarse así mismo en la atención, el sentido rítmico y la independencia de grupo (GALDÓN, 2013).

En síntesis, la práctica del canto coral es una estrategia idónea para el desarrollo integral de los alumnos de la etapa escolar básica, ya que "los sentimientos, las tradiciones, quedan reflejadas en el contenido musical y semántico de las canciones” (SERRANO y GIL, 2003), resaltándose además que con tal práctica se propicia la interdependencia de desarrollo cognitivo, afectivo y social (TORRES MORILLO; FIGUEROA; GARCÍA, 2013).

\section{El desarrollo afectivo en la práctica del canto coral}

En cuanto al desarrollo afectivo, se reconoce que la práctica del canto coral favorece la expresividad y la formación de hábitos tales como la constancia, la colaboración, la tolerancia y el autocontrol, los cuales son de suprema importancia en el desarrollo integral de todo ser humano (ZULUETA JARAMILLO, 2008). Esa integralidad vincula lo afectivo con lo cognitivo porque no puede haber desarrollo intelectual sin desarrollo afectivo (GONZÁLEZ REY, 2000; PIAGET, 2005). Estos autores consideran que no habría modificación de estructura cognitiva alguna si, al mismo tiempo, no se producen modificaciones en los sentimientos y las emociones que componen los procesos afectivos que impulsen el desarrollo intelectual.

La afectividad es considerada por Piaget (1972/1973) como una actividad reguladora de la actividad cognitiva ya que, por ejemplo, lo afectivo regula o administra la voluntad y el deseo de aprender, convirtiéndose en la energía que mueva a la acción (PIAGET, 2005). Es decir, por ejemplo, cuando se da un proceso de aprendizaje, mediante la asimilación y la acomodación 


\section{APRENDIZAJE DEL CANTO CORAL EN LA EDUCACIÓN PRIMARIA}

deviene la satisfacción. Esta relación motiva el interés por examinar los procesos afectivos que se activan durante el aprendizaje del canto coral.

\section{La voz del niño y la niña en la educación musical y el canto}

La canción como fusión de música y lenguaje es el vehículo ideal para percibir de forma natural el poder de comunicación afectiva que tiene la música al transformar en sonido nuestro mundo interior. El lenguaje oral en el sentido musical, no sólo es un componente del canto, sino que posee también sus propias posibilidades de expresión a través del timbre, del ritmo, de la altura y del significado del texto. Por ende, "la voz en el canto, en su doble vertiente de lenguaje y canto, es el instrumento expresivo y comunicativo por excelencia" (BUSTOS, 2003; p. 48), siendo "el instrumento vocal es un mecanismo maravillosamente complejo y el canto un proceso psicomotor donde se combinan: la percepción de la altura del sonido, la memoria tonal y la coordinación vocal"' (HERNÁNDEZ, 2010, p. 2).

Al cantar se da un proceso psicomotor que incluye entre otros aspectos, la percepción y la memoria de los distintos tonos o alturas de los sonidos. La pericia necesaria para dominar cada uno de estos aspectos que pudiesen determinar un canto afinado, amerita de un entrenamiento adecuado, desde los primeros años, lo que nos sugiere el ideal de la práctica del canto desde la niñez. Así que, debido a que los sujetos de este estudio fueron niños y niñas de entre 9 y 12 años de edad, es importante conocer que en sus voces prácticamente no hay diferencias por sexo hasta los años de la pubertad. "La voz infantil se parece a la voz femenina, pero con menos extensión que esta” (MATOS, 1992, p. 25). Esto determina los alcances en cuanto a los sonidos agudos o graves que con la voz se pueden alcanzar.

Por eso según Díaz (2007), a menudo se concibe a las voces infantiles como voces blancas, ya que en esta edad no se aprecian diferencias entre niñas y niños en cuanto a la altura de los sonidos que estos pueden producir al cantar. En ese sentido, Díaz (2007) recomienda que desde los primeros instantes la niña y el niño descubran su voz, con la ayuda del profesor o profesora de música, mediante juegos vocales progresivos adecuados a sus posibilidades vocales.

Ejercitando el canto el niño puede aprender las relaciones entre los sonidos, pues según Habermeyer (2002) ellos aprenden a cantar entonados a medida que practican reiteradamente esta actividad. Con el tiempo ellos llegarán a ser capaces de ver una pieza musical y cantarla 


\section{PROCESOS COGNITIVOS Y SOCIO AFECTIVOS IMPLICADOS EN EL APRENDIZAJE DEL CANTO CORAL EN LA EDUCACIÓN PRIMARIA}

con tono perfecto sin la ayuda de instrumento alguno. En este sentido Rusinek (2003) afirma, que la actividad musical es básicamente mental y que los procesos cognitivos implicados en primer lugar no son conceptuales, sino específicamente auditivos, lo que hace presumir que los estímulos auditivos pudieran desencadenar un proceso de activación para la comprensión de la música, no en conceptos musicales o teoría de la música sino en percepción musical; en ello está implicada una Pedagogía de la Comprensión (VILLEGAS, 2006). Basados en este supuesto, el inicio del proceso cognitivo en el aprendizaje de la música estaría determinado por la percepción, es decir, “[...] como la recepción y la elaboración en los centros superiores, de los datos proporcionados por los órganos de los sentidos" (Wertheimer apud BETÉS 2000, p. 120).

Además de la percepción, otras capacidades musicales asociadas con la cognición en la ejecución del canto coral son las siguientes: (a) la cognición auditiva, habilidad para apreciar el sonido como fenómeno acústico; (b) la cognición en la ejecución, expresada en la capacidad de emitir música hacia el entorno mediante la voz o los instrumentos y; (c) la cognición compositiva, vista como la capacidad de creación específicamente musical.

Con los anteriores planteamientos no se pretende agotar que los procesos mentales, aunque se considera que los mismos son suficientes para comprender la inteligencia musical, como un marco de análisis del enorme conjunto de procesos mentales puestos en juego en el aprendizaje de la música (RUSINEK, 2003).

\section{Método}

\section{Contexto de la parte empírica del estudio}

El estudio se llevó a cabo en una escuela pública de educación primaria ubicada en un área periurbana de la capital de uno de los estados venezolanos situados en la parte norte central del país. El barrio donde está situada la escuela está conformado por viviendas populares y la mayoría de los padres y madres de los alumnos ejercen oficios de la economía informal o son amas de casa.

Para la realización del trabajo se contó con la autorización tanto de los padres o madres de los estudiantes como con la del equipo directivo de la institución y de la Junta Directiva del Consejo Comunal del sector. 


\section{APRENDIZAJE DEL CANTO CORAL EN LA EDUCACIÓN PRIMARIA}

\section{Estrategia para Generar información de interés para el estudio}

Fueron organizadas 20 sesiones de clase destinadas al ensayo y práctica del canto coral. La colecta de la información se realizó en dos etapas. I Etapa (Inicial): Fue un primer encuentro comunicativo con los niños para explicarles el proceso que vivirían durante las sesiones. La explicación sobre los objetivos del estudio y la importancia del mismo, así como el valor de su colaboración fue dada también a los representantes de los niños, lo que le dio formalidad al proceso de recolección de información.

II Etapa (Inmersión profunda): Se aplicaron los instrumentos, a partir de las primeras sesiones y filmaciones de los ensayos de la coral, para los cuales, la investigadora previamente había seleccionado dos canciones para enseñarlas a los niños, considerando anticipadamente tanto la letra como la complejidad melódica de cada una de las canciones en función de la edad de los alumnos. Previo calentamiento y luego de cada sesión de ensayo, se fue realizando progresivamente el recuerdo estimulado.

Las sesiones de clase fueron filmadas en su totalidad desde la entrada al aula hasta la finalización de la sesión. La investigadora, paralelamente hacia anotaciones en su cuaderno de notas sobre algunos incidentes que ella consideraba relevantes para el estudio. Al finalizar cada sesión la investigadora luego de despedir a los niños, escribía un relato lo más detallado que le era posible sobre algunos pormenores de la actividad en función de los comportamientos de los alumnos antes, durante y después de la sesión.

La investigadora ampliaba sus registros con base en las anotaciones hechas durante la clase, las cuales en ocasiones sólo eran palabras claves que le permitieron activar la idea del momento vivido, luego ya desocupada, las ampliaba. Esto sucedía pues no resultaba nada fácil escribir anotaciones extensas cuando durante las sesiones, estaba dirigiendo las prácticas o realizando los diálogos con los alumnos.

\section{Sujetos del Estudio}

En el trabajo de campo correspondiente al estudio estuvieron directamente comprometidos, por una parte, la investigadora (primera autora de este artículo) quien es profesora especialista en Educación Musical y, por la otra, diez (10) alumnos (7 niñas y 3 niños de entre 9 y 12 años de edad) cursantes de los grados 4to, 5to y 6to. Estos alumnos fueron 
escogidos con base en su disposición a participar, manifestación de interés, responsabilidad y compromiso con la actividad. Con este grupo de alumnos se conformó un Coro de diez integrantes.

\section{Técnicas}

Diálogo e intercambio informal. Esta técnica se aplicó para conversar con los representantes al inicio de la investigación. Fue desarrollada por la maestra quien, en su rol de investigadora procuró crear un espacio para comunicar, sensibilizar y comprometer a la familia de los niños a favor de la investigación.

Observación Participante: La investigadora, simultáneamente, ejerció el rol de profesora de los estudiantes, esto le permitió tener acceso directo a las informaciones de interés para el estudio pues, dada su condición de promotora y facilitadora de la actividad pudo estar en contacto directo y vivencial con los sujetos de investigación. Esto se hizo durante los ensayos del grupo coral, lo cual permitió la indagación y valoración de los procesos de aprendizaje durante las prácticas corales, observando detalladamente los gestos y la conducta de cada niño o niña con la finalidad de gestar un clima cálido, de confianza y de placer por la actividad que estaba siendo realizada. Esto propició la comunicación permanente entre los niños y la maestra.

Mediación Cognitiva. Esta técnica fue implementada con el propósito de desarrollar y activar los procesos cognitivos de los niños. La información colectada quedó expuesta en las expresiones que los niños fueron manifestando durante los diálogos que sostuvieron con la maestra. La emergencia de dichos procesos fue propiciada por la actividad mediadora que, intencionalmente, llevó a cabo la maestra en los diálogos con los alumnos los cuales fueron registrados en grabaciones de audio y video.

Lluvia de ideas. Esta técnica sirvió para generar un ambiente relajado y así realizar interrogatorios colectivos o diálogos con preguntas generadoras hechas a propósito por la maestra para que los niños y niñas pudiesen dar respuestas, plantear ideas y exponer posibles soluciones a los problemas que sobre el aprendizaje del canto coral tuvieron lugar. Es así como se pudo crear un ambiente relajado, sin tensiones, donde los niños y niñas tuvieron la oportunidad de expresar sus planteamientos sobre las dificultades que se les iban presentando para aprenderse las canciones. 
Sesiones de Recuerdo Estimulado: En esta técnica se recurrió al uso de grabaciones de video o de audio mientras el niño o niña realizaba una tarea cantoral, en este caso se procuró hacer acercamientos de cámara a situaciones individuales y en otros casos grupales durante los diálogos o la práctica coral. Posteriormente, estas grabaciones fueron utilizadas como un estímulo para facilitar la recuperación de la información Posteriormente, estas grabaciones eran proyectadas a los alumnos con la finalidad de estimularles a recordar lo que había sucedido durante la sesión y a expresar sus impresiones, emociones, sensaciones y otras manifestaciones socio-afectivas y cognitivas.

Entrevistas no estructuradas: Estas fueron conversaciones entre los alumnos y la profesora quien, mediante preguntas puntuales solicitaba información adicional de interés para el estudio. La flexibilidad de esta técnica permitió la introducción de breves comentarios sobre cada tópico que se iba abordando. La investigadora fue apuntando algunas interrogantes que le fueron sirviendo de guía al momento de entrevistar al niño o niña, lo que se hizo, dependiendo de cada situación.

\section{Instrumentos}

Cuaderno de Notas de la Investigadora: Registro de cada experiencia, anotaciones pormenorizadas durante el periodo de recolección de datos, narraciones escritas de información relevante acerca de lo que la investigadora fue observando, viviendo, sintiendo y pensando durante el trabajo de campo.

Guías de entrevistas: elaboradas por la investigadora antes de realizar las sesiones de recuerdo estimulado, para puntualizar las preguntas sobre los fenómenos observados durante las clases que consideró de interés.

Cuestionario: elaborado por la maestra y aplicado a las madres de los niños y niñas en la charla sensibilizadora, con la intención de conocer las ideas y concepciones que ellas tenían sobre el tema de la música y el canto en la educación y formación de sus hijos.

Producción escrita: que se define en este caso, como una tarea que la maestra asignó a los niños, para conocer los pasos que cada uno realizó en aprender una canción a dos voces, utilizando como recurso un Compact Disk (CD) contentivo del canto realizado por la maestra e instrucciones habladas por ella. 


\section{APRENDIZAJE DEL CANTO CORAL EN LA EDUCACIÓN PRIMARIA}

Grabaciones de audio y video; a las cuales se le consideró un instrumento valioso que aportó gran parte de la información posteriormente analizada en este estudio.

\section{Procedimiento para analizar la información}

Primeramente, se recurrió a profesionales expertos para pasar todas las filmaciones a formato de CD. Luego, se realizaron las transcripciones de las sesiones de clase y la sesión de la charla con las representantes de los niños y niñas, tal cual sucedieron, observando cada video y transcribiendo todo lo acontecido durante la clase: los diálogos con los niños, los cantos, los ejercicios realizados.

También fueron transcriptas todas las observaciones realizadas por la investigadora, los relatos consignados en su cuaderno de notas, los comentarios, las respuestas de los representantes al cuestionario aplicado durante la charla inicial de sensibilización, la tarea (producción escrita) elaborada por los niños y niñas sobre los pasos que dieron para aprender una canción diseñada a dos voces (1era y 2da Voz), la cual fue dada a cada niño y niña en formato de cd/audio con indicaciones habladas por la maestra.

Luego de transcripta toda la información derivada de las observaciones, entrevistas, diálogos y registros de las sesiones de recuerdo estimulado, organizada en una matriz de análisis, se realizó un proceso de lectura minuciosa para ir destacando, subrayando los identificadores y formulando los indicadores que permitieron realizar la categorización o clasificación descriptiva de los procesos de aprendizaje del canto, tanto cognitivos, como afectivos, las mediaciones cognitivas que la maestra realizó, las herramientas mediacionales que ella fue utilizando durante el proceso, las fases o etapas del proceso.

Luego de ello, dichos datos fueron tratados a partir de la técnica de análisis de contenido mediante el cual todos los detalles y expresiones de nuestros informantes que quedaron asentados en los registros, fueron organizados e interpretados, lo que permitió detectar la presencia o ausencia de algunas características del contenido y hacer un recuento de datos secundarios referidos al fenómeno del canto coral en la escuela primaria surgiendo de dicho análisis los resultados, las conclusiones y recomendaciones respectivas. Es de hacer notar, que a medida que estaban siendo revisadas las notas crudas, se fueron obteniendo indicios que permitieron inferir que los procesos cognitivos y los socio-afectivos estaban entrelazados, por ello, en muchos de los casos se hizo imposible separarlos.

Revista RBBA $\mid$ Revista Binacional Brasil Argentina 


\section{Resultados}

\section{Fases en el aprendizaje del canto coral}

A partir del estudio realizado puede conjeturarse que el aprendizaje del canto coral se da por fases, genéricamente denominadas Fase Inicial, Fase Intermedia y Fase Final.

La fase inicial del aprendizaje del canto coral, se caracteriza por una Mediación sensibilizadora y diagnóstica por parte de la maestra, cuya intención es proporcionar un contexto general sobre el canto coral, sensibilizar a los alumnos hacia la práctica cantoral e indagar o revisar las condiciones musicales que tenía cada participante, además de familiarizarlos con todos los elementos que forman parte de la práctica coral. Luego se pasa a la Mediación concientizadora y activadora, donde la maestra comenzó a mediar activamente sobre los procesos cognitivos que se iban dando, para conocerlos e ir haciendo conscientes a los propios niños y niñas de sus propios procesos y así ir facilitando los aprendizajes.

En la fase intermedia los aprendizajes son propiciados por la Mediación conflictivaintegradora, dirigida a que los alumnos actúen sobre sus procesos, y proponiendo tareas más complejas para propiciar conflictos cognitivos que les indujeran a pensar en lo que no están haciendo bien y puedan hacerlo mejor para darse cuenta de cómo aprendían a cantar, para hacerlos conscientes de su aprendizaje y de todos los elementos que ponen en práctica para lograrlo.

Los aprendizajes en la fase final, son propiciados a través de la Mediación reforzadora y evaluadora, direccionada a reflexionar sobre la práctica coral, reforzar los conocimientos adquiridos y evaluar el trabajo realizado.

\section{Procesos Cognitivos y Socio-Afectivos en el Aprendizaje del Canto Coral}

El aprendizaje del canto coral en la educación primaria promueve y a la vez, dialécticamente, es promovido por la activación de variados procesos en las dimensiones cognitiva, afectiva y social de los alumnos. A continuación, serán expuestos algunos de ellos asociándolos con la respectiva fase de aprendizaje.

En la fase inicial del aprendizaje del canto coral, los procesos cognitivos que son activados por los niños son observar con atención, imitar, memorizar, describir y conceptualizar 


\section{PROCESOS COGNITIVOS Y SOCIO AFECTIVOS IMPLICADOS EN EL APRENDIZAJE DEL CANTO CORAL EN LA EDUCACIÓN PRIMARIA}

cada proceso, hacer analogías por asociación y comparación de los modos de expresión; a pesar de las dificultades iniciales para concentrarse, tiene sentido pensar que los procesos afectivos que se dieron en esta primera fase como el sentir (por parte de los niños) que el canto les produce felicidad, el ver y sentir el coro como un espacio para compartir, considerar que el canto les permite expresar sentimientos de confianza y solidaridad al ver la coral como una familia, todos estos procesos afectivos parecen haber impactado favorablemente para que a pesar de las dificultades que se les fueron presentando, los niños hayan querido mantenerse en los aprendizajes.

En la fase intermedia, a través de una Mediación conflictiva-integradora, se activaron los siguientes procesos cognitivos: observación con atención, imitar con discriminación, hacer memoria, asociación de las voces a experiencias previas, desenvolver el reconocimiento y discriminación de voces a través de una mejor concentración y su posterior evaluación; siendo este último de mucha importancia, dado que el ya percibirse capaces de evaluar su trabajo se interpreta como un avance significativo en los aprendizajes sobre el canto coral.

En cuanto a los procesos afectivos activados durante esta fase se observaron los siguientes: respeto por el trabajo, pues dejaron de reírse por lo que hacían, sentido de colaboración y solidaridad con el grupo, satisfacción por los progresos, persistencia ante las demandas, confianza en el esfuerzo para lograr la meta y sentido de pertenencia a un grupo, con mucha responsabilidad y compromiso, lo cual favoreció que el coro se constituyera como un espacio para compartir y desarrollar afectos.

En la fase final se evidenciaron procesos cognitivos asociados con una percepción auditiva más afinada; la disposición de atender con plenitud lo cual facilitaba la concentración; una memorización más activa; la capacidad de comparar y distinguir su propia voz con las de los otros miembros del coro, y así evaluar y comprender mejor los tonos. Evidenciándose también, el desarrollo de importantes procesos afectivos en los niños que les permitieron reconocer el valor del esfuerzo y la constancia, practicar la solidaridad, socializar experiencias gratas, reconocer el trabajo de la maestra quien a su vez reconoce el trabajo de los niños. Además, se manifestaron evidencias que permitieron concebir la coral como un espacio para perder el miedo escénico y desarrollar la confianza.

A continuación, se ofrece una caracterización genérica de estos procesos junto con una expresión ilustrativa extraída de exposiciones de los propios estudiantes que participaron en el 


\section{APRENDIZAJE DEL CANTO CORAL EN LA EDUCACIÓN PRIMARIA}

estudio; para ello, se identificarán los testimonios, según procedan: si son de los alumnos con la simbología de NN, y para la profesora con PM.

\section{Fase Inicial}

\section{Procesos Cognitivos}

Observar con atención: Al escuchar la canción, el ritmo de la misma, los tonos, las letras y las señas que la profesora expresaba para orientar los niños y las niñas. NN. Bueno profesora jle prestaba atención pues! ¡La escuchaba y la veía y así me iba conociendo y memorizando la canción!

Imitación. Cada uno de los niños y las niñas, procuran replicar lo que hace la profesora, tratar de replicar la forma como ella expresa los sonidos, los ritmos, identificando el nivel del tono y ritmos. NN. En realidad creo que me estaba aprendiendo la canción recordando lo que usted hizo y tratando de imitarla a usted.

Memorización: En este caso, la niña expresa que lo logra porque tiene buena memoria. Y que logra aprender algo mejor cuando le gusta, que es el caso. NN. Bueno profe no se!, porque memorizo, tengo retentiva, cuando me gusta algo me pongo insistente y me lo aprendo.

Describir y conceptualizar: En este caso, se observa que la niña, proporciona una descripción que permite conceptualizar lo que percibe, identificando si son sonidos cortos o largos. Identifica el sentido estético de la voz, otorgándole la conceptualización de bonito, diferentes, similar, etc., con los cuales, se aprecia cómo van aprendiendo a distinguir las características y el concepto de lo que significa el canto coral. NN. Cantar es como hacer sonidos, que sean bonitos maestra, que sean así. Agradables, para uno expresar amor. Algo que lo hace a uno hace sentir bien, así. una forma de hablar con música.

Analogía (asociación- comparación): Cada uno la asocian con alguna experiencia análoga o que le permite otorgar un sentido. NN. Maestra ¿puedo hablar? Las cuerdas vocales son como una liga como usted dice y así son las cuerdas del cuatro, uno mueve la clavija hacia un lado y se van escuchando más arriba y si se aflojan, se ponen aguadas y suenan bajas. 

APRENDIZAJE DEL CANTO CORAL EN LA EDUCACIÓN PRIMARIA

\section{Fase Inicial}

\section{Procesos Socio-Afectivos}

Los niños y las niñas muestran gran satisfacción por estar en el grupo, pues además de cantar, perciben que están haciendo sus amistadas, con quienes se van sintiendo en confianza y solidaridad. Esto, certifica que el canto coral es un recurso muy adecuado para promover la inserción social, facilitar el trabajo en grupo y desarrollar sentido de pertenencia. Las manifestaciones socio-afectivas más resaltantes son:

Compartir y desarrollar los afectos. A medida que se daban los pequeños progresos cada uno indicaba el gusto por estar en el grupo y ser parte de esa experiencia. NN. ;Yo me siento Feliiiz! Me gusta estar con mis amigas y hacer lo que hacemos... jcantar!

Satisfacción, cada uno comunicaba el orgullo por la oportunidad que sentían haber tenido y de poder aprender algo que antes sentían que no era parte de sus habilidades: NN. Me siento alegre, porque aprendo la música, porque siento que nunca la he dominado y aquí lo hago, itengo chance para estar en música!

Confianza a medida que avanzaban en las prácticas, se apreciaba que iban obteniendo confianza en las posibilidades de hacerlo factible; asunto que antes no lo percibían de ese modo. NN. Uno se siente más confiado, como que lo apoyan a uno, pero si tengo que estar solita ahí, ¡yo me desmayo!, todavía no me atrevo pues me daría pena.

Solidaridad. Cada uno se mostraba solidario en el esfuerzo que el otro podía realizar: NN. Dale sin pena (le dicen los compañeros) ¡todos vamos a pasar!

\section{Fase Intermediaria}

\section{Procesos Cognitivos}

Ya en esta fase los progresos se iban observando, tal como muestran los testimonios en cada proceso indicado a continuación.

Observación y atención. En esta fase la atención y la observación estaban mejor direccionadas y controlada. Se apreciaba el ya control de sus esfuerzos para alcanzar la meta. NN. Escuché varias veces la canción para aprendérmela. PM. Observan a la maestra quien les indica con un gesto de la cara el momento de cada entrada entonándoles la primera frase.

Imitar con discriminación: en este proceso se percibe la comprensión de lo que ya deben hacer debido a la capacidad que van desarrollando de imitar y discriminar entre las diversas Revista RBBA Revista Binacional Brasil Argentina 
tonalidades. NN. Es que en la parte más alta hay que hacer como usted hace natural sin gritar, porque yo siento que hago mucha fuerza y así no es que usted lo hace.

Memoria: expresada en la capacidad de activarla a través del diálogo con sus recuerdos que logran traer y hacerlos presentes a través de esa memoria: NN. ¡Si es verdad!, jahhhhh si es verdad!, a mí me ha pasado que escucho una música sin voz pues (sólo la melodía) ... así como pura música que va sonando así la canción, y uno empieza así a darse cuente de que esa música yo la conozco, ¿de dónde? ¿de dónde?, y así uno le viene algo a la mente como uno viaja como a un sitio, si de repente la escuché en una fiesta, o en una casa de alguien, se le viene a uno ese momento y el lugar y entonces izaaaas!.

Asociación, objetivada con expresiones de su cotidiano vivir que les ayudan a asimilar la idea de lo que deben hacer. NN. ;aaaaah!! si! El diafragma es ese que usted decía que había que hacer fuerza, fuerza y presión como así que uno puja para ir al baño

Reconocimiento y Discriminación, en el sentido de desarrollar la habilidad para reconocer las diferencias y así discriminar y así escoger entre las voces que deben seguir: NN. hoy en el C.D. noté la diferencia de la lera y la 2 da voz, ponía el C.D. y se escucha bien las dos voces cuando hice la voz mía encima de la otra.

Concentración. Este es uno de los procesos que requiere su mejor desarrollo dentro del canto coral, por lo tanto, es muy importante como lo destaca la observación de la profesora. PM. Los niños comenzaron a cantar con la maestra. La ven por momentos y también recurren a la lectura. En general se observa que van entonando bien, se ven concentrados en lo que están haciendo.

Evaluación, desenvolver este proceso significa un gran avance, al ser capaces de saber las mejoras y el esfuerzo que aún deben impulsar. NN. Me parece que no se escuchó muy bien. Todavía falta ensayar. Es que todavía nos confundimos. Porque algunos no se concentran en lo que están haciendo.

\section{Fase Intermediaria}

\section{Procesos Socio-Afectivos}

En esta fase intermedia, en cuanto al desarrollo de procesos socio-afectivos, se percibe la identidad del grupo. El sentido del grupo como unidad, pues todos buscan ayudarse, organizarse y hacerse responsables como miembros en el sentido de saber que todos cuentan. 


\section{PROCESOS COGNITIVOS Y SOCIO AFECTIVOS IMPLICADOS EN EL APRENDIZAJE DEL CANTO CORAL EN LA EDUCACIÓN PRIMARIA}

Respeto, este se potenció tal como lo revela el testimonio siguiente: PM. El grupo se nota un poco más maduro en este aspecto, ya no les causa risa o rareza las vocalizaciones con vocales y palabras ofrases que les hace entonar la maestra, parece que han asumido que es un ejercicio necesario y una rutina para el ensayo de la coral. Esto se nota en la postura y la aptitud que asumen.

Colaboración, durante esta fase, todos los niños y las niñas, ahora se mostraban colaboradores en todo sentido: PM. Me ofrecieron ayuda para subir los paquetes que traía, carpetas, cuadernos y bolsas.

Solidaridad ya que todos los integrantes se muestran solidarios con los progresos de los otros. PM. los niños la aplauden y ella se sonríe (una niña del grupo), lo volvió a hacer mucho mejor, con más confianza.

Satisfacción al darse por orgullosos por sus progresos. PM. Los niños se muestran contentos y satisfechos por ver que lo lograron hacerlo bien. NN. ¡Hoy me gustó que cantamos mejor! Persistencia. Ya en esta fase se observan el sentimiento de esfuerzo al querer aprender y dominar algunas letras y voces. NN. Esta canción me gusta demasiado, bueno yo me la aprendí la letra el mismo día que nos dio el C.D. Y me ponía a cantarla y ponía después la otra vez que era la segunda voz para ver si no me equivocaba y así la repetía todos los días ponía el C.D. en las tardes después de la escuela.

Confianza. En esta fase no se niegan en hacer el intento por mejorar y reconocer que aun le falta dedicarse más. NN. Bueno, profesora puedo intentarlo, porque si le soy sincero, tuve demasiados compromisos estos días con las tareas, la sinfónica, la flauta, mi profesor es muy exigente y no tuve tiempo de escucharla tanto, una vez nada más y no me la aprendí, pero lo puedo intentar

Pertenencia a un grupo, la cual se hace evidente a través del siguiente testimonio: $P M$. Una niña tomó el control y expresó que debían quedarse en sus lugares y nombró los primeros alumnos que debían hacer la primera entrada; otros, la segunda entrada y el resto y la maestra, hicieran la tercera entrada.

Responsabilidad y compromiso, todos en esta fase, ya se observan conscientes de las responsabilidades que precisan garantizar al ser parte de un grupo coral. NN. Yo vengo al próximo ensayo, hay que venir..., bueno, y además que es un compromiso con usted, mi mamá siempre me lo dice, no podemos faltar.! 

APRENDIZAJE DEL CANTO CORAL EN LA EDUCACIÓN PRIMARIA

\section{Fase Final}

\section{Procesos Cognitivos}

Percepción auditiva. Ya en esta fase, se evidencia que han logrado mayor percepción auditiva, pues apenas la profesora emite el primer sonido, ellos logran guiarse y saber qué es lo que deben hacer, tal como se indica en el siguiente testimonio: "Solamente escuchando el primer sonido nos guiamos maestra. No hace falta que cante todo". NN. Yo escucho tan clarito, que ellos en esa parte en vez de subir bajan. Yo los escucho. Y eso que yo voy cantando lo mío. Ellos son los que se equivocan.

Atención. Esta se constata en la capacidad de prestar la atención a todos los gestos y expresiones que hace la profesora investigadora, esta vez como directora del canto y que lo hacen en armonía entre ellos, de quienes están haciendo y deben hacer la misma voz; asunto fundamental en el canto coral. NN. Yo opino que hay que verla siempre. Estar pendiente de usted porque si uno se distrae se pierde y sale mal lo que estamos cantando.

Concentración en el sentido, que cada quien ha asumido su rol, sabe cuál es su papel, cuál es la voz que debe llevar a lo largo del canto coral del momento. El testimonio siguiente lo revela: "Yo la escucho a ella y, ahora, le veo la boca diciendo otra cosa y yo sigo con lo mío. Antes no la podía mirar”. Elemento sustancial para actuar dentro de un grupo coral.

Memoria. En este caso ya poseen la memoria que les permite reaccionar adecuadamente de manera casi automática, tal como lo indican los siguientes testimonios: NN. Cada quien ya se sabe su parte... Uno se la sabe bien ya ¿para qué vamos a perder tiempo pasándola cada voz? Comparar y distinguir. En este proceso ya pueden hacer comparación y asaciones con asuntos de la vida cotidiana para lograr distinguir lo que están interpretando, tal como se indica en el siguiente testimonio: "Los matices son como adornos maestra, como colores. Así como clarito, más oscuro y así, pero con la voz"; procesos éstos vitales desarrollados para poner actuar en un grupo coral. NN. Maestra es que cuando lo hacemos rápido, no se entiende ¿y si lo hacemos más lento para que se entienda la letra? No se entiende lo que decimos.

Evaluación. Los niños y las niñas, ahora, en esta fase, se dan cuenta y se permiten evaluar que ya dominan más las habilidades para cantar, pues no necesita esforzarse mucho como lo dice uno: "Ya nos salió más bonita. Yo ya no me confundo como antes. No tengo que estar tan pendiente, me sale natural.", lo cual evidencia un nivel de madurez que se percibe las debilidades pasada: "Si en realidad de ahorita, de este momento, a como empezamos, se nota 
la diferencia. Ya cada quien está más pilas.” Como queriendo decir, ahora ya somos hábiles para realizar esta actividad de poder cantar en una coral.

Comprender. En este proceso se expresa la capacidad que han desarrollado. Ya todos saben cuál es su papel en la coral. Comprende que rol tiene cada uno, tal como se expresa una alumna: "Profesora, que todos debemos cantar a un mismo nivel asiii, como que, si es piano, todospiano, que si es forte, todos forte...", lo cual muestra la capacidad de haber subjetivado el proceso para luego transferirlo al mundo objetivo, en el sentido de llevar la composición oral en función de la voz que se debe ejecutar.

\section{Fase Final}

\section{Procesos Socio-Afectivos}

Agradecimiento, lo cual se hace presente a través de objetos y regalos que le hacen llegar a la profesora en muestra de la gratitud por el trabajo realizado: PM. Una de las madres manifestó a la profesora de música su agradecimiento por preparar a su hijo para el Festival, al igual que otra mamá de otro niño, quien le llevó a la maestra como regalo un cuadro pintado por ella, como muestra de agradecimiento.

Confianza, la cual en esta fase ya se percibe plenamente desarrollada. PM. Bueno, lo que pasa es que ahora Uds., les pregunta la profesora, ¿han adquirido más confianza y más seguridad, verdad juan?

Solidaridad, la cual se constata en el siguiente testimonio: $P M$. Ante de la necesidad de hacer presentaciones en público y de cómo puede ir vestidos. Unos plantean que: NN... debemos ver que todos tengamos pantalón negro, si no ayudar a conseguirle al que no tenga para que no gaste...

Satisfacción y orgullo, estos sentimientos eran evidentes en todos los participantes del grupo, si bien los reconocimientos eran para solo dos de los integrantes del grupo coral. PM. Se mostraban satisfechos pues la maestra había pedido a dos niños que trajeran sus premios como ganadores del Festival para que contaran su experiencia y mostraran a sus amigos y amigas el reconocimiento a su trabajo musical

Esfuerzo y la constancia, se ven recompensadas con los reconocimientos obtenidos fuera del grupo coral: PM. Se empiezan a obtener logros dos miembros de la coral resultan ganadores del 2 do y ler Lugar en el Festival "Santiaguito de Oro” de la escuela. 
Todos los procesos desenvueltos en las fases anteriores se muestran en el Cuadro 1, como una síntesis integradora de todos dichos procesos activados durante las diferentes fases del aprendizaje del canto coral y sus respectivos tipos de mediación.

\begin{tabular}{|c|c|c|c|}
\hline $\begin{array}{c}\text { FASE DEL } \\
\text { APRENDIZAJE }\end{array}$ & TIPO DE MEDIACIÓN & $\begin{array}{c}\text { PROCESOS } \\
\text { COGNITIVOS }\end{array}$ & $\begin{array}{c}\text { PROCESOS } \\
\text { SOCIO-AFECTIVOS } \\
\end{array}$ \\
\hline \multirow[b]{2}{*}{ INICIAL } & $\begin{array}{c}\text { Mediación } \\
\text { sensibilizadora y } \\
\text { diagnóstica }\end{array}$ & \multirow{2}{*}{$\begin{array}{c}\text { Observar con atención } \\
\text { Imitación y } \\
\text { memorización } \\
\text { Describir y conceptualizar } \\
\text { Analogía (asociación- } \\
\text { comparación) }\end{array}$} & \multirow{2}{*}{$\begin{array}{c}\text { Compartir y desarrollar los } \\
\text { afectos } \\
\text { Satisfacción } \\
\text { Confianza } \\
\text { Solidaridad }\end{array}$} \\
\hline & $\begin{array}{c}\text { Mediación } \\
\text { concientizadora y } \\
\text { activadora }\end{array}$ & & \\
\hline INTERMEDIA & $\begin{array}{l}\text { Mediación conflictiva- } \\
\text { integradora }\end{array}$ & $\begin{array}{l}\text { Observación y atención } \\
\text { Imitar con discriminación } \\
\text { Memoria Asociación } \\
\text { Reconocimiento y } \\
\text { Discriminación } \\
\text { Concentración } \\
\text { Evaluación }\end{array}$ & $\begin{array}{c}\text { Respeto } \\
\text { Colaboración } \\
\text { Solidaridad } \\
\text { Satisfacción } \\
\text { Persistencia } \\
\text { Confianza } \\
\text { Pertenencia a un grupo } \\
\text { Responsabilidad y } \\
\text { compromiso }\end{array}$ \\
\hline FINAL & $\begin{array}{c}\text { Mediación Reforzadora y } \\
\text { evaluadora }\end{array}$ & $\begin{array}{c}\text { Percepción auditiva. } \\
\text { Atención } \\
\text { Concentración } \\
\text { Memoria } \\
\text { Comparación y distinción } \\
\text { Evaluación } \\
\text { Comprensión }\end{array}$ & $\begin{array}{c}\text { Agradecimiento } \\
\text { Confianza } \\
\text { Solidaridad } \\
\text { Satisfacción y orgullo } \\
\text { Esfuerzo y constancia }\end{array}$ \\
\hline
\end{tabular}

Cuadro 1. Síntesis de los procesos cognitivos y afectivos del canto coral organizados según las fases del aprendizaje y los tipos de mediación

\section{Conclusiones y recomendaciones}

Al terminar este trabajo se pudo constatar la importancia de promover los procesos cognitivos y socioafectivos para la constitución de un grupo de canto coral, donde sus integrantes puedan desarrollar habilidades que les permita transformar sus vidas, lo cual en este caso fue evidente, con el reconocimiento recibido por algunos de ellos por su desempeño en otro grupo coral.

Así mismo, haber podido realizar un registro pormenorizado de las actividades en la constitución de la coral, mediante el cual se puede percibir los progresos que son alcanzados por sus protagonistas, proporciona aportes tanto para profesores de música que ejerzan en la 


\section{PROCESOS COGNITIVOS Y SOCIO AFECTIVOS IMPLICADOS EN EL APRENDIZAJE DEL CANTO CORAL EN LA EDUCACIÓN PRIMARIA}

educación primaria, básica o fundamental y de investigadores interesados en promover el aprendizaje del canto coral, asunto facilitado a través del reporte metodológico conducido durante el recorrido del estudio.

Por su parte, en relación con sus beneficios como recurso de inserción social se constatan en los niveles de satisfacción alcanzados para la fase final de las 20 sesiones de trabajo realizadas, con expresiones como sobre el trabajo en equipo, acerca de la solidaridad que caracteriza la dinámica de relaciones de unos con otros; en ver lo que es más conveniente para la mayoría, y también en la confianza que sienten y por la cual ya se autorizan a comunicarse de que son y se hacen parte de una coral.

\section{REFERENCIAS}

BETÉS, Mariano. Fundamentos de Musicoterapia. Madrid: Editorial Morata. 2000.

BUSTOS SÁNCHEZ, Inés. La Voz, la técnica y la expresión. Barcelona: Editorial Paidotribo. 2003.

CABRELlES SAGREDO, $\mathrm{M}^{\mathrm{a}}$ Soledad. Las emociones y la música. Revista de Folklore. Tomo 27b. Núm. 324, 2007. Disponível em: http://www.cervantesvirtual.com/obra-visor/lasemociones-y-la-musica/html/ [Acceso el: 13 de febrero de 2015].

CROWTHER, Duane. Enseñando Técnicas y Herramientas que Ayudarán a su coro lucir Maravillosamente. E.E.U.U: Cedar Fort. 2005.

DÍAZ GÓMEZ, Maravilla. Aportaciones teóricas y Metodológicas a la Educación Musical. Barcelona: Grao. 2007.

FONZAR, Jair. Piaget: do egocentrismo (História de um conceito). Educ. rev., Curitiba, n. 5, p. 81-103, dec. $1986 . \quad$ Disponible en http://www.scielo.br/scielo.php?script=sci_arttext\&pid=S0104-40601986000100006\&lng= en\&nrm =iso [Acceso el: 30 de enero de 2014].

GALDÓN, Miguel Educación Musical. 2013. Disponible en http://www.cenoposiciones.com/docs/files/2013_edmus_15_publicidad.pdf [Acceso el: 13 de diciembre de 2015].

GONZÁLEZ, Fredy Enrique. Las Ciencias Cognitivas como Contexto para Interpretar las Nuevas Concepciones acerca del Aprendizaje. Paradigma, Vol. XX, № 2, diciembre de 1999 / 1-21. Disponible en: http://revistaparadigma.online/ojs/index.php/ paradigma/article/view/233/231. [Acceso el: 13 de febrero de 2020].

GONZÁLEZ REY, Fernando. El lugar de las emociones en la constitución social de lo psíquico: El aporte de Vigotski, Educacao \& Sociedade 21 (70), 132-148. 2000. Disponible en: 


\section{PROCESOS COGNITIVOS Y SOCIO AFECTIVOS IMPLICADOS EN EL APRENDIZAJE DEL CANTO CORAL EN LA EDUCACIÓN PRIMARIA}

https://www.scielo.br/scielo.php?pid=S0101-73302000000200006\&script=sci_abstract\&tlng $=$ pt [Acceso el: 13 de febrero de 2020].

GRINSPUN SIGUELNITZKY, Noemí; POBLETE LAGOS, Carlos. Aprendizaje musical y funciones cognitivas. Perspectivas desde la neurociencia y la cognición corporizada. Revista NEUMA. Vol. 2, 11. págs. 114-131, 2018. Disponible en: https://dialnet.unirioja.es/servlet/articulo?codigo=7168500 [Acceso el: 18 de abril de 2020].

HABERMEYER, Sharlene. Cómo estimular con música la inteligencia de los niños. México: Selector. 2002.

HERNÁNDEZ, María Dolores. El canto en las escuelas infantiles de la Comunidad de Madrid: Un recurso poco utilizado en la educación integral del niño. Revista Iberoamericana de

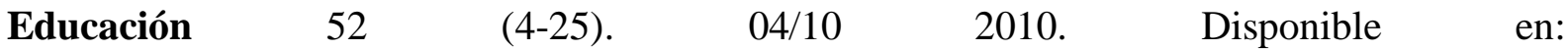
http://www.rieoei.org/deloslectores/3559Hernández.pdf [Acceso el: 16 de junio de 2014].

ZULUETA JARAMILlO, Alejandro. El Método Kodaly en Colombia. Bogotá: Editorial Pontificia Universidad Javeriana. 2008.

LUCATO, Marco (2001). El método Kodaly y la formación del Profesorado de Música Revista de la Lista Electrónica Europea de Música en la Educación. nº 7, págs. 1, 2 y 5 Disponible en: http://musica.rediris.es/leeme/ [Acceso el: 26 de marzo de 2020].

MATOS, Rebeca. Educación Musical Conocimientos Básicos para el Docente. Venezuela: FEDUPEL. 1992.

MENDOZA, Alfredo. La música, el canto y la escuela. Correo del Maestro [Revista en Linea], 61. 2001. Disponible: http://www.correodelmaestro.com/aj [Acceso el: 18 de junio de 2019].

MORÁN MARTÍNEZ, María Concepción "Psicología y Música: Inteligencia musical y desarrollo estético". Revista Digital Universitaria.1 de noviembre de 2009, Vol. 10, No. 11 Disponible en: <http://www.revista.unam.mx/vol.10/num11/art73/int73.htm\#a>. ISSN: 16076079. [Acceso el: 18 de abril de 2020].

PEREIRA, Éliton; VASCONCELOS, Miriã. O PROCESSO DE SOCIALIZAÇÃO NO CANTO CORAL: UM ESTUDO SOBRE AS DIMENSÕES PESSOAL, INTERPESSOAL E COMUNITÁRIA. Revista Música Hodie, v. 7, n. 1, 7 nov. 2007. Disponible en: https://www.revistas.ufg.br/musica/article/view/1763 https://doi.org/10.5216/mh.v7i1.1763 [Acceso el: 18 de abril de 2020].

PÉREZ SÁNCHEZ, Luz; BELTRÁN LLERA, Jesús. Dos décadas de «inteligencias múltiples»: implicaciones para la psicología de la educación. Papeles del Psicólogo, vol. 27, núm. 3, septiembre-diciembre, pp. 147-164 Consejo General de Colegios Oficiales de Psicólogos Madrid, España. 2006. Disponible https://www.redalyc.org/pdf/778/77827304.pdf. [Acceso el: 18 de abril de 2020]. 


\section{PROCESOS COGNITIVOS Y SOCIO AFECTIVOS IMPLICADOS EN EL APRENDIZAJE DEL CANTO CORAL EN LA EDUCACIÓN PRIMARIA}

PEREZ-ALDEGUER, Santiago. El canto coral: una mirada interdisciplinar desde la educación musical. Estud. Pedagóg., Valdivia, v. 40, n. 1, p. 389-404, 2014. Disponible en https://scielo.conicyt.cl/pdf/estped/v40n1/art23.pdf [Acceso el: 14 de abril de 2020].

PIAGET, Jean. Inteligencia y Afectividad. Buenos Aires: Aique.2005.

PIAGET, Jean. Problemas de Psicología Genética. Rio de Janeiro: Forense. 1973.

RODRÍGUEZ, Raquel; FERNÁNDEZ, Maximina. Desarrollo cognitivo y aprendizaje temprano: la lengua escrita en la educación infantil. Universidad de Oviedo: Servicio de Publicaciones. 1997.

RUSINEK, Gabriel. El aprendizaje musical como desarrollo de procesos cognitivos. Revista de Ciencias de la Educación, vol 19, 49-62, 2003. Disponible en http://rodin.uca.es:8081/xmlui/bitstrea [Acceso el: 12 de diciembre de 2019].

SERRANO, Montserrat; GIL, Jesús. Música. Vol. III. España: Editorial Mad S.L. 2003.

SISTEMA EDUCATIVO BOLIVARIANO. Diseño Curricular de Venezuela: CENAMEC. 2007.

TORRES MORILLO, Moraima, FIGUEROA, Neyilse, GARCÍA, Marina, Interdependencia del desarrollo cognitivo y afectivo. Aproximaciones desde la epistemología genética para la educación inicial. Revista de Pedagogía Vol. 34-35 (95-96), 59-87. 2013. Disponible en: https://www.redalyc.org/articulo.oa?id=65932613002. [Acceso el: 10 de marzo de 2020].

VILLEGAS, Ma. Margarita Pedagogía para la comprensión. Un modelo didáctico para propiciar la inclusión social. Revista de Pedagogía, vol. XXVII, núm. 79, mayo-agosto, 2006, pp. 307-350. Universidad Central de Venezuela, Caracas, Venezuela. Disponible en: https://www.redalyc.org/pdf/659/65907905.pdf [Acceso el: 15 de enero de 2014].

VILLEGAS, Margarita; GONZÁLEZ, Fredy. Línea de Investigación en Ciencias Cognitivas (LINCICOG) Paradigma, Vol. XXIV, N. ${ }^{\circ}$ 2, diciembre de 2003 /01-03. Disponible en: http://revistaparadigma.online/ojs/index.php/paradigma/article/view/302/300. [Acceso el: 14 de junio de 2019]. 


\section{SOBRE OS AUTORES}

Digna la Rosa es Profesora de la Especialidad de Educación Musical de la Universidad Pedagógica Experimental Libertador - UPEL (Maracay, Venezuela). Maestría en Educación, Mención Orientación. Profesora de Música la Escuela U.E.E. "Jorge Semidey", edo. Aragua. Violinista de la Orquesta Sinfónica de Aragua - Venezuela.

Dirección Electrónica: dignalarosa.cantocoral@gmail.com https://orcid.org/0000-0002-2322-9284

María Margarita Villegas. Doctora en Educación por la Universidade Federal Rural do Semi Árido (UFERSA; Mossoró, Brasil). Profesora Visitante en el Programa de Pós-Graduação de Ensino (POSENSINO) administrado en asociación entre las universidades UERN-UFERSAIFRN de estado de Rio Grande do Norte, Brasil.

Dirección Electrónica: margaritavillega@ hotmail.com http://orcid.org/0000-0002-4965-2291

Fredy Enrique González. Investigador acreditado en el Programa de Postgrado en Educación (PPGEd); coordinador del Grupo de Estudios de Narrativas de Profesores en Formación (GENPROF) e investigador convidado del Grupo de Estudios de la Complejidad GRECOM) de la Universidade Federal do Rio Grande do Norte (UFRN, Brasil).

Dirección Electrónica: fredygonzalezdem@gmail.com http://orcid.org/0000-0002-8079-3826

Recebido em: 21/04/2020

Aprovado em: 01/06/2020

Publicado em: 01/07/2020

\section{Notas}

\footnotetext{
${ }^{\mathrm{i}}$ http://www.corosdealava.com/que-es-un-coro/ Acceso el: 13 de febrero de 2020.
} 